\title{
On Some Properties of Spectral Radius for Brualdi-Li Matrix
}

\author{
Mandi Chen* \\ School of Mathematics and Computational Science, Sun Yat-sen University Guangzhou, 510275, P. R. China
}

Received: 4 Nov. 2013, Revised: 9 Feb. 2014, Accepted: 10 Feb. 2014

Published online: 1 May 2014

\begin{abstract}
Let $B_{2 m}$ denote the Brualdi-Li matrix,and let $\rho\left(B_{2 m}\right)$ denote the spectral radius of Brualdi-Li matrix. We obtain some properties of $\rho\left(B_{2 m}\right)$.
\end{abstract}

Keywords: Brualdi-Li Matrix,Spectral Radius,Reducible Matrix, Tournament Matrix

\section{Introduction}

A tournament matrix of order $n$ is a $(0,1)$ matrix $T$ satisfying the equation $T+T^{t}=J-I$, where $J$ is the all ones matrix, $I$ is the identity matrix, and $T^{t}$ is the transpose of $T$. The tournament matrices are inspired in the round robin competitions. Tournament matrices(and their generalizations)appear in a variety of combinatorial applications (e.g., in biology, sociology, statistics, and networks).

Brualdi and Li matrix of order $2 m$ is defined by

$$
\mathscr{B}_{2 m}=\left(\begin{array}{cc}
U_{m} & U_{m}^{t} \\
I+U_{m}^{t} & U_{m}
\end{array}\right)
$$

where $U_{m}$ is strictly lower triangular tournament matrix(all of whose entries below the main diagonal are equal to one). A matrix $A$ of order $n$ is said to be a reducible matrix if there exists a permutation matrix $P$ such that

$$
P A P^{t}=\left(\begin{array}{cc}
A_{1} & A_{3} \\
0 & A_{2}
\end{array}\right),
$$

where $A_{1}$ and $A_{2}$ are square (non-vacuous), or if $n=1$ and $A=O$. A matrix is called irreducible matrix if it is not reducible. The spectral radius of a matrix $A_{n \times n}$ defined as $\rho(A)=\max \left\{\left|\lambda_{1}\right|,\left|\lambda_{2}\right|, \cdots,\left|\lambda_{n}\right|\right\}$, where $\lambda_{1}, \lambda_{2}, \cdots, \lambda_{n}$ are eigenvalues of $A_{n \times n}$. If an nonnegative matrix $A$ is irreducible and it has exactly one eigenvalue of modulus $\rho(A)$, then the matrix is called a primitive matrix. Obviously,Brualdi and Li matrix $\mathscr{B}_{2 m}(m \geq 2)$ is primitive matrix.
In 1983 Brualdi and Li conjectured that the maximal spectral radius for tournaments of order $2 m$ is attained by the Brualdi-Li matrix [1]. This conjecture has recently be confirmed in [2]. The several interesting properties of Brualdi-Li matrix are studied. In this paper we investigate some properties of spectral radius for Brualdi-Li Matrix.

\section{Preliminaries}

The notation and terminology used in this paper will basically follow those in [3].

Let $\mathbf{1}_{m}=(1,1, \cdots, 1)_{m \times 1}^{t}, \mathbf{0}_{m}=(0,0, \cdots, 0)_{m \times 1}^{t}$, and

$$
U_{m}=\left(\begin{array}{ccccc}
0 & 0 & 0 & \cdots & 0 \\
1 & 0 & 0 & \cdots & 0 \\
\vdots & \vdots & \ddots & \vdots & \vdots \\
1 & 1 & \cdots & 0 & 0 \\
1 & 1 & \cdots & 1 & 0
\end{array}\right)_{m \times m}
$$

where $m \geq 2$ is an integer.

Lemma 2.1[3] Let $n$ be a nonnegative integer,and $A$ be a primitive matrix of order $n$. Then

$$
\lim _{k \rightarrow \infty}\left(\frac{A}{\rho}\right)^{k} \mathbf{1}_{n}=S
$$

where $\rho=\rho(A)>0, S>0$ is a eigenvector of $A$ corresponding to the eigenvalue of $\rho(A)$.

\footnotetext{
*Corresponding author e-mail: c-mandi@163.com
} 
Let $b(2 m, k)=\mathbf{1}_{2 m}^{t} \mathscr{B}_{2 m}^{k} \mathbf{1}_{2 m}$, $b_{l}(2 m, k)=\mathbf{1}_{2 m}^{t} \mathscr{B}_{2 m}^{k}\left(\begin{array}{l}\mathbf{1}_{m} \\ \mathbf{0}_{m}\end{array}\right)$, and $b_{r}(2 m, k)=\mathbf{1}_{2 m}^{t} \mathscr{B}_{2 m}^{k}\left(\begin{array}{l}\mathbf{0}_{m} \\ \mathbf{1}_{m}\end{array}\right)$,then $b(2 m, k+1)=\mathbf{1}_{2 m}^{t} \mathscr{B}_{2 m}^{k+1} \mathbf{1}_{2 m}=\mathbf{1}_{2 m}^{t} \mathscr{B}_{2 m}^{k}\left(\begin{array}{c}(m-1) \mathbf{1}_{m} \\ m \mathbf{1}_{m}\end{array}\right)$ $=m b(2 m, k)-b_{l}(2 m, k)$ $=(m-1) b(2 m, k)+b_{r}(2 m, k)$.

It is easy to verify the following result.

Lemma 2.2 Let $k, m \geq 2$ be an integer,and $\rho=\rho\left(\mathscr{B}_{2 m}\right)$. Then

(1) $\lim _{k \rightarrow \infty} \sqrt[k]{b(2 m, k)}=\rho ;$
(2) $\lim _{k \rightarrow \infty} \frac{b(2 m, k)}{b(2 m, k-1)}=\rho ;$
(3) $\lim _{k \rightarrow \infty} \frac{b_{l}(2 m, k)}{b(2 m, k)}=m-\rho ;$
(4) $\lim _{k \rightarrow \infty} \frac{b_{r}(2 m, k)}{b(2 m, k)}=\rho-m+1 ;$
(5) $\lim _{k \rightarrow \infty} \frac{b_{l}(2 m, k)}{b_{r}(2 m, k)}=\frac{m-\rho}{\rho-m+1}$.

Let $\mathscr{B}_{2 m}^{k}=\left(\begin{array}{ll}B_{11}^{(k)} & B_{12}^{(k)} \\ B_{21}^{(k)} & B_{22}^{(k)}\end{array}\right)$, and

$b_{i j}(2 m, k)=\mathbf{1}_{m}^{t} B_{i j}^{(k)} \mathbf{1}_{m}, i, j=1,2$,

where $B_{11}^{(k)}, B_{12}^{(k)}, B_{21}^{(k)}, B_{22}^{(k)}$ are matrices of order $m$.

Now that $b(2 m, k+1)=\mathbf{1}_{2 m}^{t} \mathscr{B}_{2 m}^{k+1} \mathbf{1}_{2 m}$ $=\mathbf{1}_{2 m}^{t} \mathscr{B}_{2 m}^{k}\left(\begin{array}{c}(m-1) \mathbf{1}_{m} \\ m \mathbf{1}_{m}\end{array}\right)$

$=(m-1)\left(b_{11}(2 m, k)+b_{21}(2 m, k)\right)$

$+m\left(b_{12}(2 m, k)+b_{22}(2 m, k)\right)$,

$b(2 m, k+1)=\mathbf{1}_{2 m}^{t}\left(\mathscr{B}_{2 m}^{t}\right)^{k+1} \mathbf{1}_{2 m}$

$=\mathbf{1}_{2 m}^{t}\left(\mathscr{B}_{2 m}^{t}\right)^{k}\left(\begin{array}{c}m \mathbf{1}_{m} \\ (m-1) \mathbf{1}_{m}\end{array}\right)$

$=m\left(b_{11}(2 m, k)+b_{12}(2 m, k)\right)$

$+(m-1)\left(b_{21}(2 m, k)+b_{22}(2 m, k)\right)$,

we have

$b_{11}(2 m, k)=b_{22}(2 m, k)$.

$b(2 m, k+2)=\mathbf{1}_{2 m}^{t} \mathscr{B}_{2 m}^{k+2} \mathbf{1}_{2 m}$

$=\left(m \mathbf{1}_{m}^{t},(m-1) \mathbf{1}_{m}^{t}\right) \mathscr{B}_{2 m}^{k}\left(\begin{array}{c}(m-1) \mathbf{1}_{m} \\ m \mathbf{1}_{m}\end{array}\right)$

$=2(m-1) m b_{11}(2 m, k)+m^{2} b_{12}(2 m, k)$

$+(m-1)^{2} b_{21}(2 m, k)$.

Leading to the following result.

Lemma 2.3 Let $k, m \geq 2$ be an integer. Then

(1) $\left(\begin{array}{c}b(2 m, k) \\ b(2 m, k+1) \\ b(2 m, k+2)\end{array}\right)$

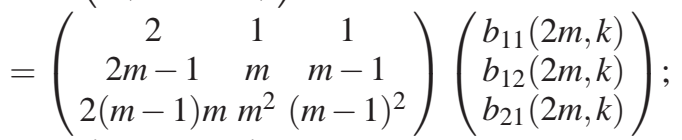

(2) $\left(\begin{array}{l}b_{11}(2 m, k) \\ b_{12}(2 m, k) \\ b_{21}(2 m, k)\end{array}\right)$

$=\left(\begin{array}{ccc}-(m-1) m & 2 m-1 & -1 \\ (m-1)^{2} & -2 m+2 & 1 \\ m^{2} & -2 m & 1\end{array}\right)\left(\begin{array}{c}b(2 m, k) \\ b(2 m, k+1) \\ b(2 m, k+2)\end{array}\right)$.
Lemma 2.4 ([4]) Let $m \geq 2$ be an integer, $\rho=\rho\left(\mathscr{B}_{2 m}\right)$, and

$\left(v_{1}, v_{2}, \ldots, v_{m}, w_{1}, w_{2}, \ldots, w_{m}\right)^{t}$ be a eigenvector of $\mathscr{B}_{2 m}$ corresponding to the eigenvalue of $\rho\left(\mathscr{B}_{2 m}\right)$, where $\sum_{i=1}^{m} v_{i}+\sum_{i=1}^{m} w_{i}=1$. Then

(1) $\rho=m-\sum_{i=1}^{m} w_{i}=m-1+\sum_{i=1}^{m} v_{i}$;

(2) $v_{m}=\frac{\rho+1-m}{\rho+1}$;

(3) $w_{k}-v_{k}=\frac{1-v_{k}(2 \rho+1)}{\rho+1}$ and

$v_{k}=\frac{1}{2 \rho+1}-\frac{2\left(\rho+\frac{1-m}{2}\right)^{2}+(1-m)\left(\frac{1+m}{2}\right)}{\rho(2 \rho+1)} \cdot\left(\frac{\rho+1)}{\rho}\right)^{2 k+1}$, $k=1,2, \cdots, m$.

\section{Some properties for spectral radius of Brualdi-Li matrix}

Theorem 3.1 Let $m \geq 2$ be an integer,and $\rho=\rho\left(\mathscr{B}_{2 m}\right)$.Then

(1) $\lim _{k \rightarrow \infty} \frac{b_{11}(2 m, k)}{b(2 m, k)}=-\rho^{2}+(2 m-1) \rho-m(m-1)$;

(2) $\lim _{k \rightarrow \infty} \frac{b_{12}(2 m, k)}{b(2 m, k)}=\rho^{2}-2(m-1) \rho+(m-1)^{2}$;

(3) $\lim _{k \rightarrow \infty} \frac{b_{21}(2 m, k)}{b(2 m, k)}=\rho^{2}-2 m \rho+m^{2}$.

Proof By Lemma 2.3(1),

$b_{11}(2 m, k)=-m(m-1) b(2 m, k)+(2 m-1) b(2 m, k+$ 1) $-b(2 m, k+2)$, then

$\frac{b_{11}(2 m, k)}{b(2 m, k)}=-m(m-1) \frac{b(2 m, k)}{b(2 m, k)}$

$+(2 m-1) \frac{b(2 m, k+1)}{b(2 m, k)}-\frac{b(2 m, k+2)}{b(2 m, k)}$. By Lemma 2.2(2),we have

$\lim _{k \rightarrow \infty} \frac{b_{11}(2 m, k)}{b(2 m, k)}=-m(m-1) \lim _{k \rightarrow \infty} \frac{b(2 m, k)}{b(2 m, k)}$

$+(2 m-1) \lim _{k \rightarrow \infty} \frac{b(2 m, k+1)}{b(2 m, k)}-\lim _{k \rightarrow \infty} \frac{b(2 m, k+2)}{b(2 m, k)}$

$=-m(m-1)+(2 m-1) \rho-\lim _{k \rightarrow \infty}\left(\frac{b(2 m, k+2)}{b(2 m, k+1)} \cdot \frac{b(2 m, k+1)}{b(2 m, k)}\right)$

$=-\rho^{2}+(2 m-1) \rho-m(m-1)$.

Using a similar approach,we have obtained (2)and (3).

Theorem 3.2 Let $m \geq 2$ be an integer,and $\rho=\rho\left(\mathscr{B}_{2 m}\right)$.Then

(1) $\lim _{k \rightarrow \infty} \frac{b_{l}(2 m, k)}{b_{l}(2 m, k-1)}=\lim _{k \rightarrow \infty} \frac{b_{r}(2 m, k)}{b_{r}(2 m, k-1)}=\rho$;

(2) $\lim _{k \rightarrow \infty} \frac{b_{i j}(2 m, k)}{b_{i j}(2 m, k-1)}=\rho$, for $i, j=1,2$.

Proof By Lemma 2.3(2),(3), $\lim _{k \rightarrow \infty} \frac{b_{l}(2 m, k)}{b_{l}(2 m, k-1)}$

$=\lim _{k \rightarrow \infty}\left(\frac{b_{l}(2 m, k)}{b(2 m, k)} \cdot\left(\frac{b_{l}(2 m, k-1)}{b(2 m, k-1)}\right)^{-1} \cdot \frac{b(2 m, k)}{b(2 m, k-1)}\right)$

$=(m-\rho)(m-\rho)^{-1} \rho$

$=\rho$.

Using a similar approach,we have obtained $\lim _{k \rightarrow \infty} \frac{b_{r}(2 m, k)}{b_{r}(2 m, k-1)}=\rho$ and (2).

Theorem 3.3 Let $m \geq 2$ be an integer, $\rho=\rho\left(\mathscr{B}_{2 m}\right)$, and

$\left(v_{1}, v_{2}, \ldots, v_{m}, w_{1}, w_{2}, \ldots, w_{m}\right)^{t}$ be a eigenvector of $\mathscr{B}_{2 m}$ corresponding to the eigenvalue of $\rho\left(\mathscr{B}_{2 m}\right)$, where $\sum_{i=1}^{m} v_{i}+\sum_{i=1}^{m} w_{i}=1$. Then

$$
\Sigma_{i=1}^{m} i\left(w_{i}-v_{i}\right)=(m-\rho)^{2} .
$$


Proof By Lemma 2.4,

$$
\sum_{i=1}^{m} i\left(w_{i}-v_{i}\right)
$$

$=\sum_{i=1}^{m} i\left(\frac{1-v_{i}(2 \rho+1)}{\rho+1}\right)$

$=\sum_{i=1}^{m} \frac{i}{\rho+1}-\frac{2 \rho+1}{\rho+1} \sum_{i=1}^{m} i v_{i}$

$=\sum_{i=1}^{m} \frac{i}{\rho+1}-\frac{2 \rho+1}{\rho+1} \sum_{i=1}^{m} i\left(\frac{1}{2 \rho+1}\right.$

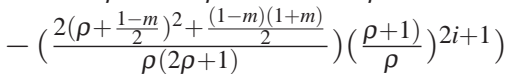

$=\frac{\left(2 \rho^{2}+2(1-m) \rho+1-m\right)}{\rho(\rho+1)} \sum_{i=1}^{m} i\left(\frac{\rho+1}{\rho}\right)^{2 i-1}$.

Now that $\sum_{k=1}^{m} k x^{k-1}=\frac{m x^{m+1}+1-(m+1) x^{m}}{(x-1)^{2}}$, thence

$\sum_{i=1}^{m} i\left(\frac{\rho+1}{\rho}\right)^{2 i-1}=\frac{\left.m\left(1+\frac{1}{\rho}\right)^{2 m+3}-(m+1)\left(1+\frac{1}{\rho}\right)^{2 m+1}+1+\frac{1}{\rho}\right)}{\left(\left(1+\frac{1}{\rho}\right)^{2}-1\right)^{2}}$

$=\frac{\rho^{4}}{(2 \rho+1)^{2}}\left(m\left(1+\frac{1}{\rho}\right)^{2 m+3}\right.$

$\left.-(m+1)\left(1+\frac{1}{\rho}\right)^{2 m+1}+1+\frac{1}{\rho}\right)$.

We have

$\sum_{i=1}^{m} i\left(w_{i}-v_{i}\right)$

$=\frac{\left(2 \rho^{2}+2(1-m) \rho+1-m\right)}{\rho(\rho+1)}\left(\frac{\rho^{4}}{(2 \rho+1)^{2}}\right)$.

$\left(m\left(1+\frac{1}{\rho}\right)^{2 m+3}-(m+1)\left(1+\frac{1}{\rho}\right)^{2 m+1}+1+\frac{1}{\rho}\right)$

$=\frac{\rho^{3}}{2 \rho+1}\left(\frac{\left(2 \rho^{2}+2(1-m) \rho+1-m\right)}{\rho(2 \rho+1)}\right)$.

$\left(m\left(1+\frac{1}{\rho}\right)^{2 m+2}-(m+1)\left(1+\frac{1}{\rho}\right)^{2 m}+1\right)$

$=\frac{\rho^{3}}{2 \rho+1}\left(\frac{\left(2 \rho^{2}+2(1-m) \rho+1-m\right)}{\rho(2 \rho+1)}\right)\left(1+\frac{1}{\rho}\right)^{2 m-1}$.

$\left(m\left(1+\frac{1}{\rho}\right)^{3}-(m+1)\left(1+\frac{1}{\rho}\right)\right)+\frac{\rho^{2}\left(2 \rho^{2}+2(1-m) \rho+1-m\right)}{(2 \rho+1)^{2}}$

$=\frac{\rho^{3}}{(2 \rho+1)}\left(\frac{1}{2 \rho+1}-\frac{\rho+1-m}{\rho+1}\right)$.

$\left(m\left(1+\frac{1}{\rho}\right)^{3}-(m+1)\left(1+\frac{1}{\rho}\right)\right)+\frac{\rho^{2}\left(2 \rho^{2}+2(1-m) \rho+1-m\right)}{(2 \rho+1)^{2}}$

$=\frac{1}{(2 \rho+1)^{2}}\left(m+2 m \rho-\rho^{2}\right)\left(m+2 m \rho-2 \rho^{2}-2 \rho\right)$

$+\frac{\rho^{2}\left(2 \rho^{2}+2(1-m) \rho+1-m\right)}{(2 \rho+1)^{2}}$

$=\frac{1}{(2 \rho+1)^{2}}\left(4 \rho^{4}-4(2 m-1) \rho^{3}+\left(4 m^{2}-8 m+1\right) \rho^{2}\right.$

$\left.+2 m(2 m-1) \rho+m^{2}\right)$

$=\frac{1}{(2 \rho+1)^{2}}\left(4 \rho^{4}+4 \rho^{3}+\left(4 m^{2}+1\right) \rho^{2}+4 m^{2} \rho+m^{2}\right.$

$\left.-\left(8 m \rho^{3}+8 m \rho^{2}+2 m \rho\right)\right)$

$=\frac{1}{(2 \rho+1)^{2}}\left(\left(4 \rho^{2}+4 \rho+1\right) \rho^{2}+4 m^{2} \rho^{2}+4 m^{2} \rho+m^{2}\right.$

$\left.-\left(4 \rho^{2}+4 \rho+1\right) 2 m \rho\right)$

$=(m-\rho)^{2}$.

\section{References}

[1] R.A. Brualdi and Q. Li, Problem 31, Discrete Math., 43(1983) 329-330.

[2] S.W. Drury, Solution of the Conjecture of Brualdi and Li, Linear Algebra Appl., 436(2012) 3392-3399.

[3] Liu Bolian, Combinatorial matrix theory, Bejing: Science Press, 2006.

[4] J.Burk and M.Tsatsomeros, On the Brualdi-Li Matrix and its Perron Eigenspace, Electron J. Linear Algebra, 23 (2012)212230.

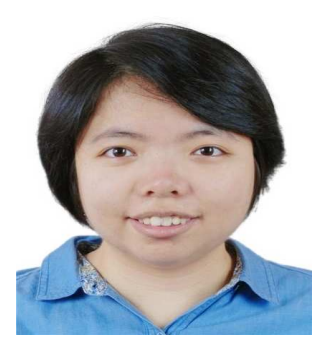

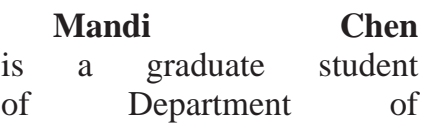
Scientific Computation and Computer Applications, School of Mathematics and Computational Science at Sun Yat-sen University. Her main research interest is mathematics. 